\title{
Avaliação quantitativa de Streptococcus do grupo mutans e Candida sp e fatores salivares na cavidade bucal de pacientes submetidos à radioterapia
}

\section{Quantitative evaluation of Streptococcus mutans and Candida sp and salivary factors in the oral cavity of patients submitted to radiotherapy}

Denise Madalena Palomari SPOLIDORIO*

Luís Carlos SPOLIDORIO*

Roberto Henrique BARBEIRO**

José Francisco HÖFLING***

Wagner Luís Carvalho BERNARDO****

Sabrina PAVAN****

\begin{abstract}
SPOLIDORIO, D. M. P.; SPOLIDORIO, L. C.; BARBEIRO, R. H.; HÖFLING, J. F.; BERNARDO, W. L. C.; PAVAN, S. Avaliação quantitativa de Streptococcus do grupo mutans e Candida sp e fatores salivares na cavidade bucal de pacientes submetidos à radioterapia. Pesqui Odontol Bras, v. 15, n. 4, p. 354-358, out./dez. 2001.
\end{abstract}

\begin{abstract}
O objetivo deste trabalho foi analisar quantitativamente os microrganismos, Streptococcus do grupo mutans e Candida $s p$, da cavidade bucal de pacientes com carcinoma de orofaringe antes, durante e após o tratamento com radioterapia e correlacionar fatores salivares como pH, capacidade tampão (CT) e fluxo salivar (FS). Amostras de saliva foram coletadas, diluídas e inoculadas em ágar SB-20 e ágar Sabouraud, respectivamente para Streptococcus do grupo mutans e Candida $s p$. Previamente à diluição, a saliva concentrada foi analisada, determinando-se os fatores salivares. Após crescimento das colônias, o número de microrganismos foi determinado em UFC $/ \mathrm{ml}$. A análise dos resultados permitiu concluir que houve correlação positiva entre os fatores salivares e a presença de microrganismos ilustrada pelo aumento no número de UFC/ml dos microrganismos analisados concomitantemente com a diminuição do fluxo salivar. Os efeitos da radiação comprometeram a homeostasia salivar e favoreceram o aumento das infecções por leveduras e bactérias durante o tratamento radioterápico.
\end{abstract}

UNITERMOS: Radioterapia; Boca; Saliva; Streptococcus mutans; Candida.

\section{INTRODUÇÃO}

O protocolo convencional para o tratamento de pacientes portadores de câncer de cabeça e pescoço é cirurgia e/ou radioterapia. A radioterapia tem como princípio a destruição de células neoplásicas malignas $^{7}$, porém também causam injúrias à população de células normais provocando efeitos colaterais e complicações variadas que afetam a cavidade bucal durante e após o tratamento. As reações agudas da radioterapia em pacientes portadores de câncer de boca geralmente ocorrem entre 10 a 17 dias após a irradiação ou mesmo na primeira semana e incluem: mucosite, alteração do paladar, perda de olfato, disfagia, otite média ou externa, anorexia, infecção bacteriana, viral ou fúngica. As reações tardias pós-radioterapia são osteorradionecrose da mandíbula, ulceração da mucosa, trismo, fibrose, necrose da mucosa, cáries dentárias, disfunção endócrina e edema de laringe ${ }^{2,14}$.

Sabe-se que o aumento da incidência da cárie é determinado pelo aumento dos Streptococcus do grupo mutans e/ou por alterações na homeostasia salivar. A incidência de Candida $s p$ na cavidade bucal e as razões para o estabelecimento da candidose são decorrentes de fatores precipitadores tais

\footnotetext{
*Professores Assistentes Doutores da Disciplina de Patologia; **Professor Assistente Doutor da Disciplina de Cirurgia; ****Alunos de Iniciação Científica - Faculdade de Odontologia de Araraquara da UNESP.

***Professor Titular da Disciplina de Microbiologia da Faculdade de Odontologia de Piracicaba da UNICAMP.
} 
SPOLIDORIO, D. M. P.; SPOLIDORIO, L. C.; BARBEIRO, R. H.; HÖFLING, J. F.; BERNARDO, W. L. C.; PAVAN, S. Avaliação quantitativa de Streptococcus do grupo mutans e Candida $s p$ e fatores salivares na cavidade bucal de pacientes submetidos à radioterapia.

Pesqui Odontol Bras, v. 15, n. 4, p. 354-358, out./dez. 2001.

como: queda de imunidade do hospedeiro, desordens endócrinas, lesões em mucosas, higiene oral deficiente, tratamento prolongado com antibióticos e corticosteróides ${ }^{17}$.

Embora existam evidências que confirmam o aumento de microrganismos na cavidade bucal de pacientes submetidos à radioterapia, alguns parâmetros envolvidos não estão adequadamente elucidados e ainda são incipientes as informações sobre a quantificação desses microrganismos, como também, no que se refere à redução salivar, e conseqüente xerostomia, por efeito da radiação usada para o tratamento. Neste trabalho, foram avaliados os microrganismos Streptococcus do grupo mutans e Candida sp, prevalentes na cavidade bucal de pacientes submetidos a radioterapia (RXt) antes, durante e após o tratamento, correlacionando-se fatores salivares como o $\mathrm{pH}$ da saliva, capacidade tampão e fluxo salivar desses pacientes.

\section{MATERIAL E MÉTODOS}

Para o presente estudo, foram analisados 11 pacientes, portadores de CEC (carcinoma espinocelular), na região de orofaringe, em tratamento na Faculdade de Odontologia de Araraquara - UNESP ou no CORA - Centro Oncológico da Região de Araraquara.

A coleta da saliva para análise dos fatores salivares - determinação da velocidade do fluxo salivar e capacidade tampão - e análise microbiológica foram realizadas antes, durante e ao término da radioterapia. Posteriormente à análise dos fatores salivares, os frascos contendo a saliva foram homogeneizados e posteriormente diluídos em série decimal de $10^{-1}$ a $10^{-4}$. Para o cultivo de Streptococcus do grupo mutans e Candida $s p$, alíquotas de cada diluição foram inoculadas respectivamente em Ágar Sacarose Bacitracina $(\mathrm{SB}-20)^{8}$ e em Ágar Saboraud Dextrose, acrescido de $0,1 \mathrm{mg} / \mathrm{ml}$ de cloranfenicol e incubadas a $37^{\circ} \mathrm{C}$ por 48 horas $^{19}$. Posteriormente ao crescimento, as colônias de ambos os microrganismos foram avaliadas por contagem das UFC/ml.

Informações sobre as formas de tratamento utilizadas, ou seja, cirurgia, radioterapia e quimioterapia acham-se descritas na Tabela 1 , assim como a dosagem (cGy) da RXt.

Para a análise dos dados, foi aplicado o teste de variância ANOVA. A análise foi complementada pelo teste de Tukey ao nível de 5\%.
TABELA 1 - Tratamento, dosagem de radiação e droga quimioterápica.

\begin{tabular}{|c|c|c|c|c|c|}
\hline \multirow{2}{*}{ Paciente } & \multicolumn{5}{|c|}{ Tratamento } \\
\hline & Cirúrgico & $\mathrm{RXt}$ & $\mathrm{Qt}$ & Dosagem RXt* & Droga Qt \\
\hline $\mathrm{P} 1$ & & $\mathrm{X}$ & & $6.600 \mathrm{cGy}$ & \\
\hline $\mathrm{P} 2$ & & $X$ & $\mathrm{X}$ & $7.240 \mathrm{cGy}$ & Cis-platina \\
\hline P3 & & $X$ & & $7.240 \mathrm{cGy}$ & \\
\hline P4 & & $X$ & & $4.500 \mathrm{cGy}$ & \\
\hline P5 & $X$ & $X$ & & $4.500 \mathrm{cGy}$ & \\
\hline P6 & & $X$ & & $7.200 \mathrm{cGy}$ & \\
\hline P7 & & $\mathrm{X}$ & $\mathrm{X}$ & $5.040 \mathrm{cGy}$ & Cis-platina \\
\hline P8 & & $\mathrm{X}$ & & $7.200 \mathrm{cGy}$ & \\
\hline P9 & & $\mathrm{X}$ & $\mathrm{X}$ & 7.200 cGy & Cis-platina \\
\hline P10 & & $\mathrm{X}$ & & $7.240 \mathrm{cGy}$ & \\
\hline P11 & $\mathrm{X}$ & $\mathrm{X}$ & & 7.240 cGy & \\
\hline
\end{tabular}

$\mathrm{RXt}=$ radioterapia; $\mathrm{Qt}=$ quimioterapia $;{ }^{*} 1 \mathrm{cGy}=1 \mathrm{rad}$.

\section{RESULTADOS}

Na Tabela 2, estão expressos os resultados obtidos das médias e desvios-padrão antes, durante e após o tratamento de radioterapia para as UFC/ml dos microrganismos Streptococcus do grupo $\mathrm{mu}$ tans e Candida $s p$, fluxo salivar, capacidade tampão e pH. Para os resultados obtidos para as variáveis fluxo salivar, capacidade tampão e pH salivar, respectivamente, observou-se que houve diminuição do fluxo salivar $(\mathrm{p}<0,05)$ no decorrer do tratamento (Tabela 2), com tendência de diminuição dos valores da capacidade tampão e pH, porém esses valores não foram estatisticamente significantes $(\mathrm{p}>0,05)$. Devido à xerostomia provocada pela irradiação, não foi possivel avaliar-se o pH e a capacidade tampão após o tratamento.

\section{DISCUSSÃO}

Aproximadamente 95\% das neoplasias da cavidade bucal são diagnosticadas histopatologicamente como sendo carcinoma espinocelular ${ }^{21}$, sugerindo-se que a associação de hábitos interligados como a utilização de fumo e álcool aumentam a probabilidade de incidência dessas neopla$\operatorname{sias}^{4,11}$, dados esses confirmados por meio da análise dos pacientes no nosso trabalho. O fluxo salivar durante e após o tratamento com radioterapia diminuiu $(\mathrm{p}<0,05)$ (Tabela 2). Esses resulta- 
SPOLIDORIO, D. M. P.; SPOLIDORIO, L. C.; BARBEIRO, R. H.; HÖFLING, J. F.; BERNARDO, W. L. C.; PAVAN, S. Avaliação quantitativa de Streptococcus do grupo mutans e Candida sp e fatores salivares na cavidade bucal de pacientes submetidos à radioterapia.

Pesqui Odontol Bras, v. 15, n. 4, p. 354-358, out./dez. 2001.

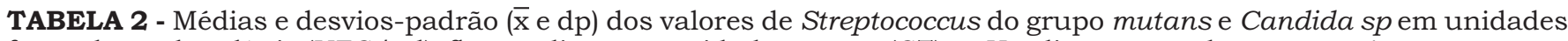
formadoras de colônia (UFC/ml), fluxo salivar, capacidade tampão (CT) e pH salivar antes, durante e após tratamento.

\begin{tabular}{l|c|c|c|c|c|c|c|c|c|c}
\hline \hline \multirow{2}{*}{ Tratamento } & \multicolumn{2}{|c|}{ UFC - Streptococcus } & \multicolumn{2}{|c|}{ UFC - Candida } & \multicolumn{2}{|c|}{ Fluxo salivar } & \multicolumn{2}{|c}{ CT } & \multicolumn{2}{c}{$\mathrm{pH}$} \\
\cline { 2 - 12 } & $\overline{\mathrm{x}}$ & $\pm \mathrm{dp}$ & $\overline{\mathrm{x}}$ & $\pm \mathrm{dp}$ & $\overline{\mathrm{x}}$ & $\pm \mathrm{dp}$ & $\overline{\mathrm{x}}$ & $\pm \mathrm{dp}$ & $\overline{\mathrm{x}}$ & $\pm \mathrm{dp}$ \\
\hline Antes & $7,4 \times 10^{5} \mathrm{a}$ & $1,22 \mathrm{a}$ & $1,2 \times 10^{4} \mathrm{a}$ & $2,54 \mathrm{a}$ & $0,74 \mathrm{a}$ & $0,53 \mathrm{a}$ & $2,8 \mathrm{a}$ & $1,7 \mathrm{a}$ & $5,4 \mathrm{a}$ & $2,85 \mathrm{a}$ \\
\hline Durante & $2,6 \times 10^{5} \mathrm{a}$ & $0,36 \mathrm{a}$ & $3,34 \times 10^{5} \mathrm{~b}$ & $105,19 \mathrm{~b}$ & $0,3 \mathrm{a}$ & $0,45 \mathrm{a}$ & $2,4 \mathrm{a}$ & $1,3 \mathrm{a}$ & $4,8 \mathrm{a}$ & $2,59 \mathrm{a}$ \\
\hline Depois & $6,5 \times 10^{5} \mathrm{a}$ & $1,38 \mathrm{a}$ & $2,33 \times 10^{5} \mathrm{~b}$ & $63,04 \mathrm{~b}$ & $0,08 \mathrm{~b}$ & $0,04 \mathrm{~b}$ & $*$ & $*$ & $*$ & $*$ \\
\hline \hline
\end{tabular}

*Coleta da saliva com "swab". Letras iguais indicam que não há diferença pelo teste de Tukey $(\mathrm{p}<0,05)$.

dos confirmam os achados obtidos por BERNHOFT; SKAUG $^{3}$ (1985). Entre a primeira e a segunda semana de tratamento, diagnosticou-se a presença de xerostomia, que é um dos primeiros sintomas causados pela radioterapia, relatado por AUGUST et al. ${ }^{1}$ (1996). Esses resultados indicam que o dano da radioterapia às glândulas salivares maiores produz uma diminuição severa no fluxo salivar dentro das duas primeiras semanas de tratamento. A xerostomia resultante da incapacidade de produzir saliva é progressiva e persistente durante a radioterapia, sendo o dano ao tecido salivar irreversível e na maioria dos casos leva o paciente a tornar-se permanentemente xerostômico ${ }^{12}$. Segundo DREIZEN et al. ${ }^{10}$ (1976) e CARL ${ }^{6}$ (1977), a saliva e fluxo salivar sofrem alterações conforme a evolução do tratamento das neoplasias pela radioterapia e/ou quimioterapia, pois estes destroem ou danificam severamente as glândulas salivares, provocando alterações como a atrofia, degeneração e fibrose dos tecidos. Especula-se, no entanto, se o dano ao tecido glandular é causado por efeitos diretos da radiação sobre as células secretoras e os ductos, ou se é secundário à lesão das delicadas estruturas vasculares, com conseqüente aumento da permeabilidade vascular, edema intersticial e infiltrações de células inflamatórias ${ }^{20}$.

Os valores de capacidade tampão e $\mathrm{pH}$, respectivamente, diminuíram ao longo do tratamento. Esses resultados estão de acordo com os trabalhos de DREIZEN et al..$^{10}$ (1976) e CARL ${ }^{6}$ (1977), os quais sugerem que a saliva residual torna-se viscosa, com menor poder de lubrificação, proteção e há uma acentuada diminuição do $\mathrm{pH}$, o que faz com que ela fique mais ácida devido às alterações eletrolíticas e à irradiação das glândulas salivares. As variações dos valores de $\mathrm{pH}$ e capacidade tampão salivares não foram estatisticamente significantes antes e durante o tratamento, como relatado também por BERNHOFT; SKANG ${ }^{3}$ (1985). Esses auto- res observaram significativas alterações quando analisaram esses itens que são próximos aos valores encontrados neste trabalho.

Nas últimas décadas, a associação entre valores de $\mathrm{UFC} / \mathrm{ml}$ de Candida e algumas patologias da cavidade bucal tem sido investigada. Considerando-se que o agente etiológico Candida é usualmente encontrado na maioria das formas de candidoses e em algumas condições menos comuns, devemos ressaltar a prevalência de Candida albicans e todas as espécies deste gênero, como microrganismos que - em pequeno número - compõem o trato digestivo e regiões mucocutâneas normais e alteradas do ser humano. Alterações na microbiota bucal ocorrem em pacientes xerostômicos. Aumento na proporção de Streptococcus e Lactobacillus, assim como acidificação do $\mathrm{pH}$ da saliva foi observado em pacientes submetidos à radioterapia $^{13}$.

No presente trabalho, constatou-se que 91\% dos pacientes eram infectados por Candida $s p$ antes de serem submetidos a tratamento pela radioterapia; porém, após o tratamento os valores de $\mathrm{UFC} / \mathrm{ml}$ de Candida $s p$ e Streptococcus do grupo mutans aumentaram. Estes dados coincidem com os resultados obtidos por BROWN et al. ${ }^{5}$ (1993), os quais observaram aumento na quantidade de Streptococcus mutans, espécies de Lactobacillus, Candida (principalmente C. albicans) e Staphylococcus, e decréscimo no número de Streptococcus sanguis, espécies de Neisseria e Fusobacterium, em pacientes submetidos à radioterapia, sugerindo que as infecções por leveduras e bactérias, comumente se desenvolvem durante o tratamento radioterápico devido a xerostomia e queda de imunidade $^{2}$.

Na Tabela 2, pode-se observar que os resultados para os microrganismos Candida foram estatisticamente significantes entre os períodos antes/durante e antes/após, detectando-se aumento 
SPOLIDORIO, D. M. P.; SPOLIDORIO, L. C.; BARBEIRO, R. H.; HÖFLING, J. F.; BERNARDO, W. L. C.; PAVAN, S. Avaliação quantitativa de Streptococcus do grupo mutans e Candida sp e fatores salivares na cavidade bucal de pacientes submetidos à radioterapia.

Pesqui Odontol Bras, v. 15, n. 4, p. 354-358, out./dez. 2001.

de microrganismos do gênero Candida na cavidade bucal desses pacientes, à semelhança dos dados relatados por ROSSIE et al..$^{18}$ (1987) e PAULA et $a l^{16}$ (1990). Os dados obtidos individualmente em relação às $\mathrm{UFC} / \mathrm{ml}$ de Candida $s p$ parecem estar de acordo com GOODMAN et al. ${ }^{12}$ (1992), os quais relatam o aumento na contagem destes microrganismos, podendo persistir por vários meses, propiciando maior suscetibilidade à candidose, devendo-se assim, realizar tratamento com antifúngicos tópicos ou sistêmicos, dependendo da gravidade da infecção, o que foi feito com os nossos pacientes. Isso explicaria o aumento desses microrganismos, mesmo que não estatisticamente significante entre os períodos durante/após, detectado em nossos resultados.

Por outro lado, observou-se uma alteração quantitativa de Streptococcus do grupo mutans na cavidade bucal dos pacientes irradiados, todavia esses valores não foram estatisticamente significantes. Uma explicação possivel para a falta de correlação entre estes microrganismos, poderia residir na baixa amostragem, pois somente três pacientes não realizaram exodontia total. Como observado por MARX; JOHNSON ${ }^{15}$ (1987), antes do início da radioterapia, deve-se realizar uma anamnese completa, verificando-se as condições dos dentes, condições socioeconômicas, culturais e o prognóstico do paciente. Pelo risco de desenvolver osterorradionecrose, em pacientes com bom prognóstico, com motivação de higienização e dentes sem lesão, devem ser mantidos os dentes na cavidade bucal. Entretanto, os pacientes que não apresentam essas características devem ser sub- metidos a exodontias. Embora DIB; CURY ${ }^{9}$ (1993) contrariamente relatem que as exodontias devem ser evitadas e preconizem o tratamento odontológico restaurador, em grande parte dos casos relatados no presente estudo foram realizadas exodontias totais dos pacientes, devido às más condições bucais existentes.

Do ponto de vista clínico, algumas considerações devem ser feitas antes do início do tratamento, ou seja, medidas profiláticas prévias à radioterapia poderiam beneficiar esse grupo de pacientes submetidos a tratamentos neoplásicos, como também minimizar o efeito da xerostomia, além de ser utilizada saliva artificial e orientação para maior consumo de água, como observado por ROSSIE et $a l .^{18}(1987)$.

\section{CONCLUSÕES}

Os resultados obtidos e a discussão estabelecida permitem concluir que:

- o tratamento com radioterapia produz uma diminuição severa no fluxo salivar dentro das duas primeiras semanas de tratamento;

- os fatores salivares estão correlacionados com o aumento dos microrganismos;

- a xerostomia pode quebrar o equilíbrio ecológico da microbiota bucal, favorecendo o aparecimento de infecções e maior proliferação de microrganismos;

- os efeitos da radiação favorecem o aumento de Candida $s p$ e Streptococcus do grupo mutans (em edentados) nos pacientes submetidos ao tratamento.

SPOLIDORIO, D. M. P.; SPOLIDORIO, L. C.; BARBEIRO, R. H.; HÖFlinG, J. F.; BERNARDO, W. L. C.; PAVAN, S. Quantitative evaluation of Streptococcus mutans and Candida $s p$ and salivary factors in the oral cavity of patients submitted to radiotherapy. Pesqui Odontol Bras, v. 15, n. 4, p. 354-358, out./dez. 2001.

The aim of this study was to quantify the microorganisms Streptococcus mutans and Candida sp in the oral cavity of patients with oropharynx carcinoma, before, during and after radiotherapy, and to correlate the results with salivary factors such as pH, buffer capacity (CT) and flow rate (FS). Saliva samples were collected, diluted and inoculated in SB-20 agar and in Sabouraud agar, for Streptococcus mutans and Candida sp, respectively. Previously to dilution, the concentrated saliva was analyzed, and the salivary factors were determined. After the growth of colonies, the number of microorganisms was determined in $\mathrm{CFU} / \mathrm{ml}$. The analysis of the results allowed to conclude that the salivary factors are related to the presence of microorganisms, and that the number of $\mathrm{CFU} / \mathrm{ml}$ increased as salivary flow rate decreased. The effects of radiation compromised salivary homeostasis and favored the increase of infection by yeasts and bacteria.

UNITERMS: Radiotherapy; Mouth; Saliva; Streptococcus mutans; Candida. 
SPOLIDORIO, D. M. P.; SPOLIDORIO, L. C.; BARBEIRO, R. H.; HÖFLING, J. F.; BERNARDO, W. L. C.; PAVAN, S. Avaliação quantitativa de Streptococcus do grupo mutans e Candida sp e fatores salivares na cavidade bucal de pacientes submetidos à radioterapia. Pesqui Odontol Bras, v. 15, n. 4, p. 354-358, out./dez. 2001.

\section{REFERÊNCIAS BIBLIOGRÁFICAS}

1. AUGUST, M.; WANG, J.; PLANTE, D. et al. Complications associated with therapeutic neck radiation. J Oral Maxillofac Surg, v. 54, n. 12, p. 1409-1415, Dec. 1996.

2. BARASCH, A.; SAFFORD, M.; EISENBERG, E. Oral cancer and oral effects of anticancer therapy. Mt Sinai J Med, v. 65, n. 516, p. 370-377, Oct/Nov. 1998.

3. BERNHOFT, C. H.; SKAUG, N. Oral findings in irradiate edentulous patients. Int $\mathbf{J}$ Oral Surg, v. 14, n. 5, p. 416-427, Oct. 1985.

4. BLOT, W. J.; MCLAUGHLIN, J. K.; WINN, D. M. et al. Smoking and drinking in relation to oral and pharyngeal cancer. Caries Res, v. 48, n. 11, p. 3282-3287, June 1988.

5. BROW, L. R.; DREIZEN, S.; HANDLER, S. et al. Effect of radiation-induced xerostomia on human oral microflora. J Dent Res, v. 54, n. 4, p. 740-750, July/Aug. 1975 .

6. CARL, W. Managing the oral manifestation of cancer therapy. Postgrad Med, v. 61, n. 1, p. 85-92, Jan. 1977.

7. DAMM, D. D.; NEVILLE, B. W.; GEISSLER, R. H. et al. Dentinal candidiasis in cancer patients. Oral Surg Oral Med Oral Pathol, v. 65, n. 1, p. 56-60, Jan. 1988.

8. DAVEY, A. L.; ROGERS, A. H. Multiple types of bacterium Streptococcus mutans in the human mouth and their intra-family transmission. Arch Oral Biol, v. 29, n. 6, p. 435-60, June, 1984.

9. DIB, L. L.; CURY, M. M. A Odontologia na Oncologia. Âmbito Hospitalar, v. 4, p. 39-44, 1993.

10. DREIZEN, S. A.; BROWN, L. R.; HANDLER, S. et al. Radiation-induced xerostomia in cancer patients. Cancer, v. 38, n. 1, p. 273-278, Jan. 1976.

11. EPSTEIN, J. B.; MARSHALL, M. F.; LE, N. D. Risk factors for oropharyngeal candidiasis in patients who receive radiation therapy for malignant conditions of the head and neck. Oral Surg Oral Med Oral Pathol, v. 72, n. 2, p. 169-174, Aug. 1993.

12. GOODMAN, J. L.; WINSTON, D. J.; GREENFIELD, R. A. et al. A controlled trial of fluconazole to prevent fungal infections in patients undergoing bone marrow transplantation. N Eng1 J Med, v. 326, n. 13, p. 845-851, Mar. 1992.

13. LLORY, H.; DAMMRON, A.; GIOANNI, M. et al. Some population changes in oral anaerobic microorganisms, Streptococcus mutans and yeasts following irradiation of the salivary glands. Caries Res, v. 6. n. 4, p. 298-311, June 1972.

14. LOPES, A. M.; COLETTA, R. D.; ALVES, F. A et al. Reconhecendo e controlando os efeitos colaterais da radioterapia. Rev Assoc Paul Cir Dent, v. 52 , n. 3, p. 241-244, maio/jun. 1998.

15. MARX, R. E.; JOHNSON, R. P. Studies in the radiobiology of osteoradionecrosis and their clinical significance. Oral Surg Oral Med Oral Pathol, v. 64, n. 4, p. 379-390, Oct. 1987.

16. PAULA, C. R.; SAMPAIO, M. C. C.; BIRMAN, E. G. et al. Oral yeasts in patients with cancer of the mouth, before and during radiotherapy. Mycopathologia, v. 112, n. 2, p. 119-124, Nov. 1990.

17. RAMIREZ-AMADOR, V.; SILVERMAN Jr., S.; MAYER P. et al. Candidal colonization and oral candidiasis in patients undergoing oral and pharyngeal radiation therapy. Oral Surg Oral Med Oral Pathol Oral Radiol Endod, v. 84, n. 2, p. 149-153, Aug. 1997.

18. ROSSIE, K. M.; TAYLOR, J.; BECK, F. M. et al. Influence of radiation therapy on oral Candida albicans colonization: a quantitative assessment. Oral Surg Oral Med Oral Pathol, v. 64, n. 6, p. 698-701, Dec. 1987.

19. SANDVEN, P. Laboratory identification and sensitivity testing of yeast isolates. Acta Odontol Scand, v. 48, n. 1, p. 27-36, Feb. 1990.

20. WHITMAYER, C. C. Radiotherapy for head and neck neoplasm. Gen Dent, v. 45, n. 4, p. 363-370, Jul./Aug. 1997.

21. WINGO, P. A.; TONG, T.; BOLDEN, S. Cancer statistics. Cancer, v. 45, n. 1, p. 8-30, Jan./Feb. 1995.

Recebido para publicação em 27/11/00 Enviado para reformulação em 07/06/01 Aceito para publicação em 24/08/01 